\title{
Expansion, geometry, and gravity
}

\author{
Robert R Caldwell ${ }^{1}$ and Marc Kamionkowski ${ }^{2}$ \\ ${ }^{1}$ Department of Physics and Astronomy, Dartmouth College, 6127 Wilder \\ Laboratory, Hanover, NH 03755, USA \\ ${ }^{2}$ Mail Code 130-33, California Institute of Technology, Pasadena, CA 91125, \\ USA \\ E-mail: Robert.R.Caldwell@dartmouth.edu and kamion@tapir.caltech.edu
}

Received 20 May 2004

Accepted 6 September 2004

Published 17 September 2004

Online at stacks.iop.org/JCAP/2004/i=09/a=009

doi:10.1088/1475-7516/2004/09/009

\begin{abstract}
In general-relativistic cosmological models, the expansion history, matter content, and geometry are closely intertwined. In this brief paper, we clarify the distinction between the effects of geometry and expansion history on the luminosity distance. We show that the cubic correction to the Hubble law, measured recently with high-redshift supernovae, is the first cosmological measurement, apart from the cosmic microwave background, that probes directly the effects of spatial curvature. We illustrate the distinction between geometry and expansion with a toy model for which the supernova results already indicate a curvature radius larger than the Hubble distance.
\end{abstract}

Keywords: supernova type Ia, classical test of cosmology, gravity

ArXiv ePrint: astro-ph/0403003 
Perhaps the most surprising implication of the general-relativistic cosmological models which first emerged roughly 80 years ago [1] was the possibility that the spatial geometry of an isotropic homogeneous Universe could be nontrivial. Alternatives to the intuitively obvious Euclidean geometry (a 'flat' Universe) include a three-sphere (the 'closed' Universe) and a three-hyperboloid (the 'open' Universe).

Over the past five years, a suite of cosmic microwave background (CMB) experiments have provided unique tests of the spatial geometry and provided very compelling evidence that the Universe is flat $[2,3]$. More precisely, the evidence is that the Universe is consistent with flat, by which we mean that if the Universe is open or closed, the radius of curvature is larger than roughly three times the Hubble distance.

As robust as the $\mathrm{CMB}$ results may be, the cosmological geometry is of such fundamental significance that it is well worth exploring alternative avenues. Inspired by the recent announcement of the measurement with high-redshift supernovae [4] indicating a transition from cosmic deceleration to acceleration, here we point out that the cubic correction to Hubble's linear relation between the luminosity distance and the cosmological redshift depends on the spatial geometry $[5,6]$. Although well appreciated by experts, the distinction between the effects of expansion and geometry is often imprecise in the literature. This probably stems from the fact that the matter content, expansion, and geometry are linked in general relativity. In particular, previous measurements that found an accelerating expansion [7] have not yet directly probed the spatial curvature ${ }^{3}$. We argue here that the measurement of the cubic correction to the luminosity-distance-redshift relation is the first significant, non-CMB cosmological test that probes the spatial geometry.

Below we first clarify the dependence of the luminosity distance on the geometry and on the expansion history. We then discuss a straw-man model (that respects general relativity but postulates an exotic form of matter) that illustrates the effects of geometry and expansion history on the cubic term. Next we show how the the quartic correction to the Hubble law might then distinguish between geometry and expansion history in this toy model. We then argue that no other classical (non-CMB) cosmological tests have yet probed the geometry.

The luminosity distance of an object at redshift $z$ is $d_{L}=(L / 4 \pi F)^{1 / 2}$, where $L$ is the luminosity for a given object (presumed known for the high-redshift supernovae being detected), and $F$ is the energy flux received from that object. The expression for the luminosity distance in a dynamic, homogeneous, isotropic spacetime is

$$
\frac{d_{L}(z)}{(1+z)}= \begin{cases}R \sinh \left[\frac{c}{H_{0} R} \int_{0}^{z} \frac{\mathrm{d} z^{\prime}}{E\left(z^{\prime}\right)}\right], & \text { open, } \\ c H_{0}^{-1} \int_{0}^{z} \frac{\mathrm{d} z^{\prime}}{E\left(z^{\prime}\right)}, & \text { flat, } \\ R \sin \left[\frac{c}{H_{0} R} \int_{0}^{z} \frac{\mathrm{d} z^{\prime}}{E\left(z^{\prime}\right)}\right], & \text { closed. }\end{cases}
$$

Here, $H_{0}$ is the Hubble constant, $c H_{0}^{-1} \simeq 4300 \mathrm{Mpc}$ is the Hubble distance, and $R$ is the (comoving) radius of curvature of the open or closed Universe; for the closed Universe, it is precisely the radius of the three-sphere, and the argument of the sine is the angle

\footnotetext{
3 To be clear, we mean to discuss the curvature of the homogeneous, isotropic spatial sections in an FRW universe, which is nonzero in a nonflat universe only, not the spacetime curvature, e.g. Ricci scalar.
} 
around the three-sphere subtended by an object at redshift $z$. Note that as $R \rightarrow \infty$, the open/closed expressions reduce to that for the flat Universe, as they should. The explicit appearance of $R$ in these equations, as well as the sin and the sinh in the closed- and open-Universe expressions, is a direct consequence of the non-Euclidean spatial geometry.

The function $E(z)$ quantifies the expansion rate as a function of redshift; it is defined from

$$
[H(z)]^{2} \equiv\left(\frac{\dot{a}}{a}\right)^{2}=H_{0}^{2}[E(z)]^{2}
$$

where $H(z)$ is the expansion rate at redshift $z, a(t)=(1+z)^{-1}$ is the scale factor at time $t$, and the dot denotes the derivative with respect to time. Equations (1) follow from the properties of the spacetime, independent of the laws of gravitation.

According to general relativity, the expansion rate is determined by the matter content and the spatial curvature. If the Universe consists of nonrelativistic matter (whose energy density scales as $a^{-3}$ ) plus a cosmological constant (whose energy density remains constant), then

$$
E(z)=\left[\Omega_{\mathrm{m}}(1+z)^{3}+\Omega_{\Lambda}+\Omega_{K}(1+z)^{2}\right]^{1 / 2},
$$

where

$$
\Omega_{\mathrm{m}}=\frac{8 \pi G \rho_{\mathrm{m}}}{3 H_{0}^{2}}, \quad \Omega_{K}=\frac{c^{2}}{\left(a_{0} H_{0} R\right)^{2}}, \quad \Omega_{\Lambda}=\frac{\Lambda c^{2}}{3 H_{0}^{2}},
$$

and $\rho_{\mathrm{m}}$ is the nonrelativistic-matter density today, $G$ is the gravitational constant, and $\Lambda$ is the cosmological constant. For reference, current best-fit values from a variety of measurements are $\Omega_{\mathrm{m}} \simeq 0.3$ and $\Omega_{\Lambda} \simeq 0.7$.

Equation (3) implies $\Omega_{\mathrm{m}}+\Omega_{\Lambda}+\Omega_{K}=1$. Thus, measurement of $\Omega_{\mathrm{m}}$ and $\Omega_{\Lambda}$ (i.e., determining the matter content of the Universe) fixes $\Omega_{K}$, even without observing any geometrical effects. This is the explicit statement that in general relativity the matter content of the Universe determines the geometry.

Let us now return to equation (1). This can be Taylor expanded (see, e.g., [6]),

$d_{L}(z)=\frac{c z}{H_{0}}\left\{1+\frac{1}{2}\left(1-q_{0}\right) z-\frac{1}{6}\left[1-q_{0}-3 q_{0}^{2}+j_{0} \pm \frac{c^{2}}{H_{0}^{2} R^{2}}\right] z^{2}+\mathcal{O}\left(z^{3}\right)\right\}$,

where \pm is + for a closed Universe and - for an open Universe. In this expression, $q_{0} \equiv-(\ddot{a} / a) H_{0}^{-2}$ (evaluated today) is the well known deceleration parameter, and $j_{0} \equiv(\dddot{a} / a) H_{0}^{-3}$ (also evaluated today) is the 'jerk', a scaled third time derivative of the scale factor $a(t)$ of the Universe. The linear term $\left(H_{0}\right)$ in this relation is the Hubble law, and the quadratic correction $\left(q_{0}\right)$ is the deceleration. These do not depend on $R$; they thus both depend only on the expansion history, and not on the spatial curvature.

The cubic term in equation (5) is the first term in the Taylor expansion that depends explicitly on the spatial curvature $R$. In other words, it is the first term in the redshift expansion in which the dependence of the brightness of an object depends on the convergence or divergence of nearly parallel light rays that occurs in a non-Euclidean spatial geometry. This is the first key point of our paper.

In a Universe that consists of nonrelativistic matter and a cosmological constant, the densities $\Omega_{\mathrm{m}}$ and $\Omega_{\Lambda}$ determine the deceleration parameter and they determine the geometry; it has thus become quite common for cosmologists to associate the cosmological 


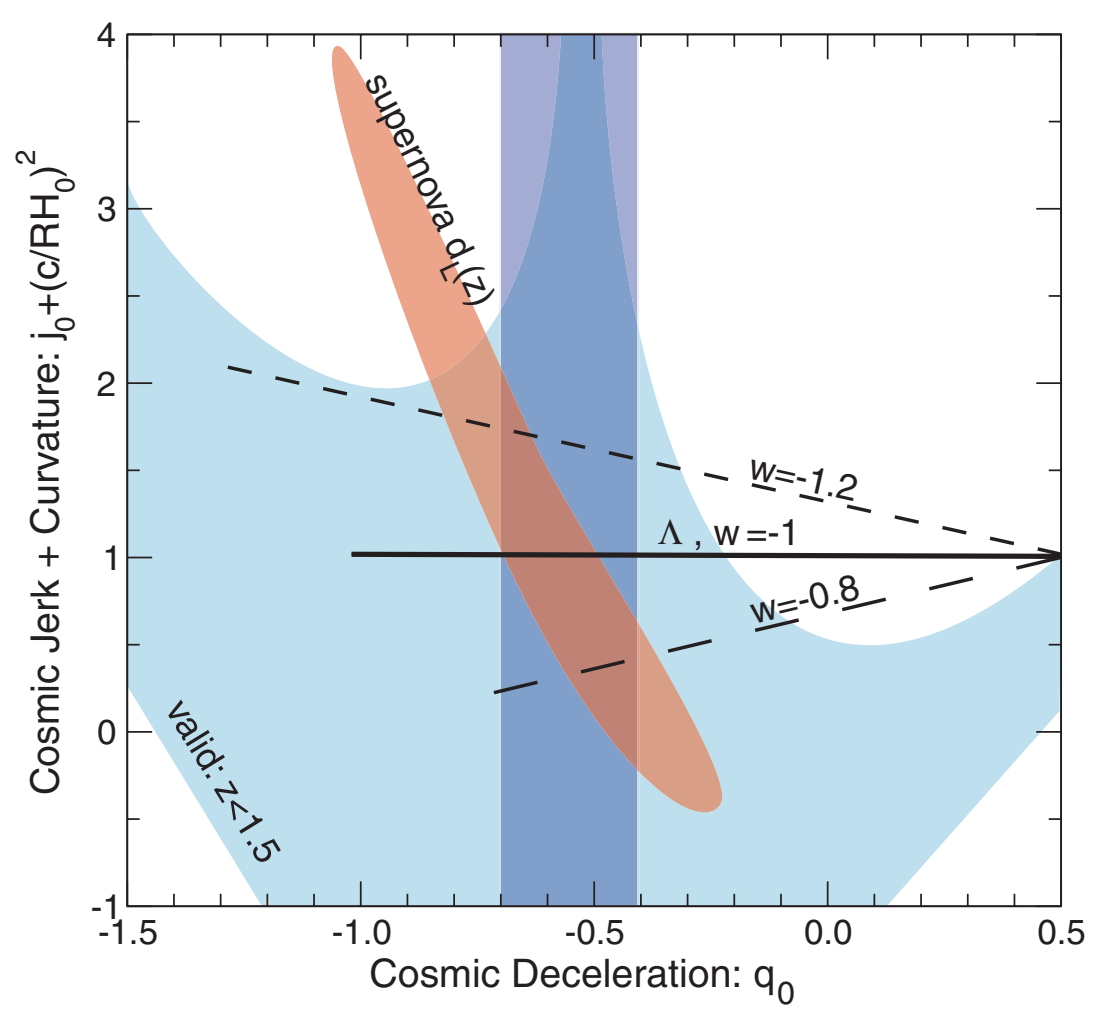

Figure 1. Current constraints to the $\left[q_{0}, j_{0}+\left(H_{0} R\right)^{-2}\right]$ plane. The dark shaded, curved ellipsoidal region is the $95 \%$ confidence-level constraint from recent highredshift supernova measurements [4]. The light-shaded region shows the domain of validity of the cubic redshift expansion; more precisely, outside these regions, there would be a unit magnitude error at $z=1.5$ introduced by the quartic term. The solid curve indicates a family of flat cosmological-constant models with decreasing matter density from right to left, terminating at $q_{0}=-1$ when $\Omega_{\mathrm{m}}=0$. The short-dashed curve shows the same for flat models with quintessence with $w=-1.2$ (i.e., a phantom-energy model $[11,12]$ ), and the long-dashed curve shows the same for $w=-0.8$. The vertical band shows the range of values for the spatially curved model with $\Omega_{\mathrm{m}}+\Omega_{\Lambda}=1$ discussed in the text and matter density spanning the range $0.2<\Omega_{\mathrm{m}}<0.4$.

expansion with the cosmological geometry. The second key point of our paper is to remark that there is indeed a distinction. For example, if the expansion dynamics were determined by some theory other than general relativity - as some have begun to speculate in view of the apparent cosmic acceleration - then the geometry would not necessarily be fixed by the expansion history. Even within the context of general relativity, they need not be linked, as the following toy model illustrates.

Consider a family of models that contain, in addition to nonrelativistic matter and a cosmological constant, some form of exotic matter with an energy density that scales as $a^{-2}$ with the scale factor $a(t)$; this could be accomplished, for example in the single-texture model [8] or with a network of nonintersecting strings [9] although the specific mechanism is irrelevant here. Suppose also that the Universe is closed. Then the Friedmann equation 
becomes

$$
[E(z)]^{2}=\Omega_{\mathrm{m}}(1+z)^{3}+\Omega_{\Lambda}-\Omega_{K}(1+z)^{2}+\Omega_{\mathrm{t}}(1+z)^{2},
$$

where $\Omega_{\mathrm{t}}=8 \pi G \rho_{\mathrm{t}} / 3 H_{0}^{2}$ is the texture energy density today. The crucial feature of this model is that the contributions to the expansion rate of exotic matter and spatial curvature evolve with the same time dependence. Therefore, if we set $\Omega_{\mathrm{t}}=\Omega_{K}$, or equivalently, $\rho_{\mathrm{t}}=3 c^{2} / 8 \pi G\left(a_{0} R\right)^{2}$, then the texture and spatial-curvature terms in the Friedmann equation cancel out. We thus arrive at a family of models that have the same $\Omega_{\mathrm{m}}$ and $\Omega_{\Lambda}$, with $\Omega_{\mathrm{m}}+\Omega_{\Lambda}=1$, and identical expansion histories, but are parametrized by a nonzero spatial curvature $R$ that we can freely dial without affecting $E(z)$. Consequently, the only influence of spatial curvature on classical tests of cosmology occurs through the geometric factor, sin/sinh in equation (1). In the redshift expansion of the luminosity distance, equation (5), the presence of spatial curvature first appears starting at the third term.

If the Universe consists of nonrelativistic matter and a cosmological constant, then general relativity gives $q_{0}=\frac{1}{2}\left(\Omega_{\mathrm{m}}-2 \Omega_{\Lambda}\right)$ and $j_{0}=\Omega_{\mathrm{m}}+\Omega_{\Lambda}$. Suppose then (consider hypothetically a CMB-data-free world), that the matter density is determined by dynamical measurements. Then $q_{0}$ would give us $\Omega_{\Lambda}$, from which we could predict $j_{0}$. Since the cubic term depends on the combination $j_{0}+c^{2} /\left(H_{0} R_{0}\right)^{2}$ (cf equation (5)), measurement of the cubic correction to the Hubble law then provides a measurement of the geometry. This is illustrated in figure 1, where current supernova constraints to the $\left[q_{0}, j_{0}+c^{2} /\left(H_{0} R_{0}\right)^{2}\right]$ parameter space are shown. Within the class of our toy models with $0.2<\Omega_{\mathrm{m}}<0.4$ and $\Omega_{\mathrm{m}}+\Omega_{\Lambda}=1$ and $R$ variable, it is clear that $R$ is restricted to be bigger than roughly the Hubble radius.

Suppose, however, that rather than a cosmological constant, the dark energy has some time variation [10] with an energy-density decay parametrized by an equation-ofstate parameter $w$. If so, then the jerk $j_{0}$ depends on $w$ as well as $\Omega_{\mathrm{m}}$ and $\Omega_{\Lambda}[5,6]$, and it thus cannot be predicted from measurements of the deceleration parameter; i.e., the jerk and geometry cannot be distinguished by the cubic correction to the luminosity distance in this broader class of models. This is illustrated by the solid, long-dashed, and shortdashed lines in figure 1 that indicate families of flat models with $w=-1$ (cosmological constant), $w=-0.8$ (quintessence), and $w=-1.2$ (phantom energy [11, 12]). In this case, one may hope to use the quartic correction to the luminosity distance [6],

$$
\frac{c z^{4}}{24 H_{0}}\left[2-2 q_{0}-15 q_{0}^{2}-15 q_{0}^{3}+5 j_{0}+10 q_{0} j_{0}+s_{0}+\frac{2 c^{2}\left(1+3 q_{0}\right)}{H_{0}^{2} R^{2}}\right],
$$

where $s_{0}=(\dddot{a} / a) H_{0}^{-4}$ is the 'snap', to isolate the spatial curvature. This is a difficult approach, however, in the absence of a theory of the dark energy. As the parameter space of cosmological models is expanded, more terms in the expansion must be accurately determined in order to isolate the effects of the geometry.

Before closing, we note that the recent supernova [4] measurement of the cubic correction to the luminosity distance is the first non-CMB empirical probe of the spatial geometry of the Universe. All classical cosmological measurements depend on some combination of the expansion history parametrized by $E(z)$ and the spatial curvature $R$ [13]. So, for example, the linear growth of perturbations depends only on the expansion history. The number-count-redshift relation, Alcock-Paczynski test, and angular-diameter-redshift relation (determined, e.g., with radio sources) depend on both 
the expansion and geometry, but none have been determined precisely at sufficiently high redshift to tease out a cubic term. We thus conclude that the new supernova measurements are the first non-CMB measurements to probe directly the effects of nontrivial spatial geometry.

\section{Acknowledgments}

This work was supported at Caltech by NASA NAG5-11985 and DoE DE-FG03-92ER40701, and at Dartmouth by NSF grant PHY-0099543.

\section{References}

[1] Bernstein J and Feinberg G, 1989 Cosmological Constants: Papers in Modern Cosmology (New York: Columbia University Press)

[2] Kamionkowski M, Spergel D N and Sugiyama N, 1994 Astrophys. J. Lett. 426 L57

[3] Miller A D et al, 1999 Astrophys. J. Lett. 524 L1 de Bernardis P et al, 2000 Nature 404955 [SPIRES]

Hanany S et al, 2000 Astrophys. J. Lett. 545 L5

Halverson N W et al, 2002 Astrophys. J. 56838 [SPIRES]

Mason B S et al, 2003 Astrophys. J. 591540 [SPIRES]

Benoit A et al, 2003 Astron. Astrophys. 399 L2 [SPIRES]

Goldstein J H et al, 2003 Astrophys. J. 599773 [SPIRES]

Spergel D N et al, 2003 Astrophys. J. Suppl. 148213

[4] Riess A G et al, Type Ia supernova discoveries at $z>1$ from the Hubble space telescope: evidence for past deceleration and constraints on dark energy evolution, 2004 Preprint astro-ph/0402512

[5] Chiba T and Nakamura T, 1998 Prog. Theor. Phys. 1001077 [SPIRES]

[6] Visser M, Jerk and the cosmological equation of state, 2003 Preprint gr-qc/0309109

[7] Perlmutter S et al (Supernova Cosmology Project Collaboration), 1998 Nature 39151 [SPIRES]

Riess A G et al (Supernova Search Team Collaboration), 1998 Astron. J. 1161009 [SPIRES]

Schmidt B P et al, 1998 Astrophys. J. 50746 [SPIRES]

Garnavich P M et al, 1998 Astrophys. J. 50974 [SPIRES]

Perlmutter S et al (Supernova Cosmology Project Collaboration), 1999 Astrophys. J. 517565 [SPIRES]

Knop R A et al, 2003 Preprint astro-ph/0309368

Tonry J L et al, 2003 Astrophys. J. 5941 [SPIRES]

[8] Kamionkowski M and Toumbas N, 1996 Phys. Rev. Lett. 771332 [SPIRES]

[9] Spergel D and Pen U-L, 1997 Astrophys. J. Lett. 491 L67

[10] Caldwell R R, Dave R and Steinhardt P J, 1998 Phys. Rev. Lett. 801582 [SPIRES]

Ratra B and Peebles P J E, 1988 Phys. Rev. D 373406 [SPIRES]

Wetterich C, 1995 Astron. Astrophys. 301321 [SPIRES]

Coble K, Dodelson S and Frieman J, 1997 Phys. Rev. D 551851 [SPIRES]

Turner M S and White M, 1997 Phys. Rev. D 564439 [SPIRES]

[11] Caldwell R R, 2002 Phys. Lett. B 54523 [SPIRES]

[12] Caldwell R R, Kamionkowski M and Weinberg N N, 2003 Phys. Rev. Lett. 91071301 [SPIRES]

[13] Peebles P J E, 1993 Principles of Physical Cosmology (Princeton, NJ: Princeton University Press) 